\title{
Numerical Analysis of Microcracks of Fly Ash/Slag Concrete with Cobble as Coarse Aggregate
}

\author{
Songfang Xie $\mathbb{i}$, Mingxing Gao $\mathbb{i}$, and Hangtian Li \\ College of Energy and Transportation Engineering, Inner Mongolia Agricultural University, Hohhot 010018, China \\ Correspondence should be addressed to Songfang Xie; 275577812@qq.com and Mingxing Gao; hhhtxsf@imau.edu.cn
}

Received 28 October 2021; Revised 17 December 2021; Accepted 18 December 2021; Published 15 January 2022

Academic Editor: Bingxiang Yuan

Copyright (C) 2022 Songfang Xie et al. This is an open access article distributed under the Creative Commons Attribution License, which permits unrestricted use, distribution, and reproduction in any medium, provided the original work is properly cited.

In order to improve mechanical properties of fly ash/slag concrete with large size cobble as coarse aggregate, this paper analyzes the effect of different factors on the concrete through the flexural strength test. The Monte Carlo simulation is used in the finite element solver of ANSYS to conduct the four-point bending beam test. Three-dimensional and two-dimensional finite element models are established to discuss how the gradation of large size cobbles affects the performance of the concrete by comparing macromechanical experiments. Results show that the gradation of large size cobbles is the main factor affecting the performance of the concrete. Slag generates the least effect on the concrete with cobble as coarse aggregate. When the mixing amount of slag and fly ash is $10 \%$, the concrete presents the best flexural performance. Through the numerical loading test of the two-dimensional model for fly ash/slag concrete with cobble as coarse aggregate, it can be concluded that the change of the concrete follows the law of macromechanical properties.

\section{Introduction}

Building rural roads is an important part of China's economy. In recent years, China has witnessed rapid expansion of roads. Rural roads, as key part of China's road network, link urban and rural areas. Therefore, rural road construction has great significance. The construction should be environmentfriendly and of good quality while taking into account local conditions. New materials and technologies are encouraged to use to improve the quality of the road and reduce costs. As the rural road is low type pavement, the construction technology required is not intensive and the equipment and machinery used are not advanced. In many occasions, the construction materials do not meet engineering requirements. For example, as the Hinggan League is rich in cobbles, hard and durable cobbles are used as coarse aggregate for the concrete pavement. Given that the size of these cobbles is too large but little varied, and as coarse aggregates they are seldom sieved, the roads constructed with these coarse aggregate are of low quality with short service life and weak vehicle load capacity, which raises concerns for safety and effectiveness. Currently, there are few studies on fly ash/slag concrete with cobble as coarse aggregate. Zhang et al. studied the effect of the aggregate size on the elastic modulus and bond strength of the concrete pavement transition area and established a random aggregate model with the maximum aggregate size being 20,40 , 60 , and $80 \mathrm{~mm}$. They found that the larger the aggregate size, the lower the bond strength of the transition area, and the bond strength is reduced by a larger margin [1].

Chen Yi proposed a method to evaluate the fracture performance of the concrete. A three-point bending test was carried out to measure the mixing ratio of two types of aggregates of different sizes in the concrete. It was found that concretes with different mixing ratios had similar maximum peak load $\mathrm{P}$ but different ductility under the three-point bending condition [2].

$\mathrm{Du}$ et al. discussed the effect of the maximum aggregate size of $10 \mathrm{~mm}, 20 \mathrm{~mm}, 30 \mathrm{~mm}$, and $40 \mathrm{~mm}$ on the performance of the concrete [3]. The larger the aggregate size, the lower the splitting tensile strength of the concrete.

Mozaffar et al. analyzed the effect of different aggregate sizes on the performance of high-strength concrete and found the optimal content [4]. 
Zhong et al. conducted research on the splitting tensile strength of the concrete with different sizes of coarse aggregate and proposed a modified DIF formula that took into account the strain rate effect and the size effect, which was used to predict the DIF of the concrete [5].

Liu sifted the coarse aggregate into 4 grades, namely, $9.5 \mathrm{~mm}, 16 \mathrm{~mm}, 19 \mathrm{~mm}$, and $26.5 \mathrm{~mm}$. The study found that when the aggregate size increased from $9.5 \mathrm{~mm}$ to $26.5 \mathrm{~mm}$, the slump of the concrete first increased and then decreased, and the compressive strength of the concrete gradually decreased. It was concluded that the maximum aggregate size of the concrete should not exceed $19 \mathrm{~mm}$ [6].

Hao studied the effect of the maximum aggregate size, and the single-graded [7], two-graded, and three-graded aggregate on the flexural strength of the concrete. Results showed that the larger the aggregate size, the lower the flexural strength of the concrete, and an appropriate maximum aggregate size and gradation can improve the concrete's flexural strength [8-10].

Sridhar et al. studied different nominal maximum aggregate sizes $(10 \mathrm{~mm}$ and $20 \mathrm{~mm})$ and found that the larger the nominal aggregate size, the shorter the endurance life of the concrete, and vice versa [11]. Wu studied the continuous graded crushed cobbles of $5-10 \mathrm{~mm}, 5-16 \mathrm{~mm}, 5-20 \mathrm{~mm}$, 5-25 mm, and 5-31.5 mm [12]. His study showed that, with the increase of the aggregate size, the compressive strength of concrete increased first and then decreased. It was concluded that the maximum aggregate size of crushed cobbles should not exceed $20 \mathrm{~mm}$. Dittmer and Beushausen studied the effect of the coarse aggregate content and aggregate size on the cracking of the adhesive layer through ring pressure test and found that there was no correlation [13]. Mohammed studied the effect of the maximum aggregate size (MAS) $(12.5 \mathrm{~mm}, 19.0 \mathrm{~mm}, 25.0 \mathrm{~mm}, 37.5 \mathrm{~mm}$, and $50.0 \mathrm{~mm}$ ) on ultrasonic pulse velocity (UPV) of the concrete. Based on the relationship between UPV and the compressive strength, he proposed the elastic modulus and Young's modulus for different maximum aggregate sizes of the brick [14]. Karamloo et al. studied the effect of the maximum aggregate size (dmax) on fracture parameters and brittleness of lightweight self-compacting concrete, taking into account six components with $\operatorname{dmax}=9.5,12.5$, and $19 \mathrm{~mm}$, as well as the water-cement ratio of 0.35 and 0.4 [15]. Results showed that fracture toughness and fracture energy increased with the increase of dmax, which could be explained by the change of fractal dimension. Al-Oraimi et al. studied the effect of mineralogy of the coarse aggregate on the compressive strength and crack resistance [16]. They studied two aggregate sizes $(10 \mathrm{~mm}$ and $20 \mathrm{~mm})$ and found that the maximum aggregate size of $20 \mathrm{~mm}$ was between 72.5 and $77.5 \mathrm{MPa}$. So they concluded that a smaller maximum aggregate size would result in higher strength, and the mineralogy of the coarse aggregate would affect the strength of the concrete. Han et al. studied the effects of steel fiber length and the maximum size of the coarse aggregate on mechanical properties of the steel fiber concrete [17]. They proposed the rational range of the ratio of steel fiber length to the maximum size of the coarse aggregate, which was 1.25 , and that to fracture characteristics was 14 . Kim et al. studied the effect of the coarse aggregate size on the dynamic compressive strength of the concrete [18].

In this study, a series of SHPB tests were conducted on mortar and concrete specimens at different maximum aggregate sizes. The test results showed that the larger the maximum size of the coarse aggregate, the greater the heterogeneity of the specimens. Jawahar et al. mixed coarse aggregates of different sizes and found that the mixture had little effect on the compressive strength of the self-compacting concrete [19]. With the increase of the coarse aggregate content, the splitting tensile strength of concrete increased. When $\mathrm{Vu}$ et al. applied unconfined compression to the concrete they found that the aggregate size had little effect on the strength of the concrete [20]. When high confining pressure was applied, the larger the coarse aggregate size was, the smaller the ultimate deformation strain of the concrete was.

Yuan used the finite element software PLAXIS to establish a multilevel filled slope model and compared the difference between the unreinforced slope and the reinforced slope under the same working condition [21].

At present, many scholars have proved that the concrete achieved enhanced performance through the compounding of materials $[22,23]$ and obtained the reaction principle through microscopic analysis [24, 25].

Given that the concrete used for rural road construction in the Hinggan League is of large size and single-graded, this paper studied mechanical properties of the concrete with large size cobbles as coarse aggregate, in order to improve the strength of the concrete and reduce engineering costs while using local available materials. This paper selected sustainable raw materials from industrial wastes to protect the environment. Common industrial wastes include slag and fly ash. Based on that, this paper did research on the effect of the gradation of large size cobbles and the mixing amount of slag and fly ash on the performance of the concrete and simulated the distribution of large size cobbles in concrete cracks in ANSYS.

\section{Materials}

2.1. Raw Material for the Test. The materials were as follows: cement: P.O 42.5 ordinary Portland cement produced by Ulanhot Hongcheng Cement Co., LTD, with an apparent density of $3090 \mathrm{~kg} / \mathrm{m}^{3}$; slag powder: slag powder produced by Ulanhot Hongcheng Cement Co., LTD, with an apparent density of $2602 \mathrm{~kg} / \mathrm{m}^{3}$; fly ash: fly ash produced by Ulanhot Hongcheng Cement Co., LTD, with an apparent density of $2304 \mathrm{~kg} / \mathrm{m}^{3}$. Cobbles were used as the coarse aggregate for the test with a maximum size of $40 \mathrm{~mm}$. They were divided into two grades by size: $5-20 \mathrm{~mm}$ and $20-40 \mathrm{~mm}$. The maximum aggregate size of the small cobble was $20 \mathrm{~mm}$ and that of the medium cobble was $40 \mathrm{~mm}$. The mixing ratio of the small cobble to the medium cobble was $20 \%: 80 \%, 30 \%$ : 70\%, 40\%: 60\% and 50\%: 50\%, respectively.

2.2. Hardened Concrete Properties. The concrete with cobble as coarse aggregate was cured in the standard curing room for 28 days under the temperature of $20 \pm 3^{\circ} \mathrm{C}$ and the 
humidity of $95 \%$. First, we conducted the orthogonal experiment to study the effect of three different factors on the flexural strength of the concrete with cobble as coarse aggregate. These three factors were mixing amount of fly ash (A), mixing amount of slag powder (B), and gradation of large size cobbles (C). Each factor had four levels. The flexural strength was tested by SYE-300 digital display testing machine. The orthogonal levels of various factors are shown in Table 1.

The results are shown in Table 2. The flexural strength of the concrete after curing for $28 \mathrm{~d}$ was selected as the indicator, as is shown in Table 3 ( $K$ refers to the average of the test value at a certain level, and $R$ refers to the difference between the minimum and the maximum value of the average value at a certain level).

The extreme difference reflects the effect of the factor on the flexural strength [26]. From the table, it can be seen that $R$ follows the sequence of $\mathrm{C}$ (gradation) $>\mathrm{A}$ (fly ash) $\mathrm{B}>$ (slag), indicating that the gradation of large size cobbles is the most important influencing factor for the performance of the concrete while slag presents the weakest effect. The combination of $\mathrm{A} 2 \mathrm{~B} 2 \mathrm{C} 1$ and $\mathrm{A} 1 \mathrm{~B} 3 \mathrm{C} 3$ has a flexural strength of $5.19 \mathrm{MPa}$ and $5.01 \mathrm{MPa}$, respectively, which are quite high. This is because the concrete is mixed with fly ash and slag whose shape and properties change with the curing of the concrete and that they contribute to the hardening of the concrete. With a proper amount of fly ash and slag, the concrete will experience the hydration reaction along with its curing and become strong compound that is bending resistant. Excessive or inadequate amounts of fly ash and slag will affect the hydration reaction of the concrete, resulting in lower compacting between aggregates and lower flexural strength, as is shown by the combination of A1B1C1 and A4B4C1.

Three specimens were given the four-point bending beam test, and the test results are shown in Table 2.

It can be seen from Figure 1 that the range follows the sequence of $\mathrm{C}$ (gradation) $>\mathrm{A}$ (fly ash) $\mathrm{B}>$ (slag). As the range reflects the effect of the factors on the performance of the concrete, the results indicate that the gradation of large size cobble is the most important influencing factor. The slag presents the weakest effect on the concrete.

As can be seen from Figure 2, after adding fly ash to the concrete, the flexural strength has improved. With the increase of the fly ash content, the flexural strength of the concrete after curing for $28 \mathrm{~d}$ shows a rising trend first. When the fly ash content is $10 \%$, the flexural strength of concrete reaches the maximum value; when the fly ash content is $5 \%$, the fly ash is activated. The flexural strength of the concrete with the mixing amount of 5\% is lower than that of $10 \%$. With the increase of the mixing amount, the pozzolanic reaction is initiated and reacts with the cement hydration product $\mathrm{Ca}(\mathrm{OH})_{2}$. The bond capacity between the aggregate and the cement is improved, so the flexural strength of the concrete is increased. However, when the amount of fly ash is excessive, with the consumption of $\mathrm{Ca}(\mathrm{OH})_{2}$ and the increase of fly ash, there is insufficient $\mathrm{Ca}(\mathrm{OH})_{2}$ to react with the fly ash, resulting in weaker activity of the fly ash. Therefore, when the fly ash content exceeds
$10 \%$, with the increase of fly ash content, the flexural strength of the concrete decreases gradually.

From level 1 to level 4, the flexural strength of the concrete increases first and then decreases with the increase of the slag. It reaches its maximum value at level 2 and then decreases gradually. The effect of the fly ash content on the flexural strength of the concrete is similar to that of the fly ash. The optimal slag content is $10 \%$ or level 2 . The flexural strength of the concrete at level 2 has increased by $6.5 \%$ compared with that at level 1 . As the slag is more activated, it can produce more hydration products, which will fill the gap of the cement slurry. After the concrete is mixed with the slag, large holes in the concrete will be replaced by small holes. In other words, the slag produces a microaggregate filling effect. The flexural strength of the concrete has decreased by $6.7 \%$ from level 2 to level 4 , indicating that the slag also has a limit. Excessive slag would affect the hydration reaction of the concrete, thus reducing its flexural strength.

The effect of the gradation of large size cobbles on the flexural strength has first increased and then decreased. As the gradation of large size cobbles decreases from $20 \%: 80 \%$ to $40 \%: 60 \%$, the flexural strength of the concrete increases gradually and then decreases gradually. The gradation of the coarse aggregate has certain influence on the flexural strength, and the bonding capacity between the aggregate and the cement affects the flexural strength of the concrete. When the gradation is $40 \%: 60 \%$, the flexural strength of the concrete reaches the maximum value, and the bonding capacity is the highest. Later, with the gradual decrease of the middle cobbles, the bonding capacity between the aggregate and the cement decreases, and the flexural strength of the concrete gradually decreases.

\subsection{The Four-Point Bending Beam Test. The Monte Carlo} simulation is also called stochastic simulation. It is used to estimate the random probability of the occurrence of an event or to obtain data characteristics of random variables. It can be summarized as three main steps: building or describing the probability process; sampling from the known probability distribution; conducting the estimation. This paper applied the Monte Carlo simulation to concrete specimens (four-point bending beam test) in a given area to obtain data for the location and aggregate size. Random numbers should be used as the medium to further generate the data. To study the numerical simulation of the concrete, this study used uniformly distributed variables with the probability distributions of $(0,1)$. These variables were used as random variables for the follow-up numerical simulation to obtain data for random generation and placement of aggregates in concrete specimens. In this paper, three-dimensional and two-dimensional finite element models are established for fly ash/slag concrete with cobble as coarse aggregate in order to study the distribution of large size cobbles at different gradations and the nature of stress and strain during the fracture process. In the four-point bending beam experiment, the damage was made in the middle of the beam. In order to save time and calculation, $150 \mathrm{~mm}$ in the middle of the beam was selected. After the data for the 
TABLE 1: Orthogonal levels of different factors.

\begin{tabular}{lccc}
\hline Level & \multicolumn{2}{c}{ Factors } \\
& Mixing amount of fly ash (\%) & Mixing amount of slag powder (\%) & Gradation of large size cobble (\%) \\
\hline 1 & 5 & 5 & $20 \%: 80 \%$ \\
2 & 10 & 10 & $30 \%: 70 \%$ \\
3 & 15 & 15 & $40 \%: 60 \%$ \\
4 & 20 & 20 & $50 \%: 50 \%$ \\
\hline
\end{tabular}

TABLE 2: Orthogonal experiment results.

\begin{tabular}{|c|c|c|c|c|}
\hline Number & $\begin{array}{c}\text { Mixing amount of fly ash (A) } \\
(\%)\end{array}$ & $\begin{array}{c}\text { Mixing amount of slag powder (B) } \\
(\%)\end{array}$ & $\begin{array}{c}\text { Gradation of large size cobble (C) } \\
(\%)\end{array}$ & $\mathrm{F} 28 \mathrm{~d}$ cu MPa \\
\hline $\mathrm{A} 1 \mathrm{~B} 1 \mathrm{C} 1$ & 5 & 5 & $20 \%: 80 \%$ & 4.01 \\
\hline $\mathrm{A} 1 \mathrm{~B} 2 \mathrm{C} 2$ & 5 & 10 & $30 \%: 70 \%$ & 4.34 \\
\hline A1B3C3 & 5 & 15 & $40 \%: 60 \%$ & 5.01 \\
\hline $\mathrm{A} 1 \mathrm{~B} 4 \mathrm{C} 4$ & 5 & 20 & $50 \%: 50 \%$ & 4.43 \\
\hline $\mathrm{A} 2 \mathrm{~B} 1 \mathrm{C} 2$ & 10 & 5 & $30 \%: 70 \%$ & 4.39 \\
\hline $\mathrm{A} 2 \mathrm{~B} 2 \mathrm{C} 1$ & 10 & 10 & $20 \%: 80 \%$ & 5.19 \\
\hline $\mathrm{A} 2 \mathrm{~B} 3 \mathrm{C} 4$ & 10 & 15 & $50 \%: 50 \%$ & 4.61 \\
\hline $\mathrm{A} 2 \mathrm{~B} 4 \mathrm{C} 3$ & 10 & 20 & $40 \%: 60 \%$ & 4.84 \\
\hline A3B1C3 & 15 & 5 & $40 \%: 60 \%$ & 4.86 \\
\hline A3B2C4 & 15 & 10 & $50 \%: 50 \%$ & 4.97 \\
\hline $\mathrm{A} 3 \mathrm{~B} 3 \mathrm{C} 1$ & 15 & 15 & $20 \%: 80 \%$ & 4.46 \\
\hline A3B4C2 & 15 & 20 & $30 \%: 70 \%$ & 4.65 \\
\hline $\mathrm{A} 4 \mathrm{~B} 1 \mathrm{C} 4$ & 20 & 5 & $50 \%: 50 \%$ & 4.53 \\
\hline $\mathrm{A} 4 \mathrm{~B} 2 \mathrm{C} 3$ & 20 & 10 & $40 \%: 60 \%$ & 4.42 \\
\hline $\mathrm{A} 4 \mathrm{~B} 3 \mathrm{C} 2$ & 20 & 15 & $30 \%: 70 \%$ & 4.81 \\
\hline $\mathrm{A} 4 \mathrm{~B} 4 \mathrm{C} 1$ & 20 & 20 & $20 \%: 80 \%$ & 4.00 \\
\hline
\end{tabular}

TABLE 3: Range analysis for the orthogonal experiment.

\begin{tabular}{lccc}
\hline Parameter & \multicolumn{3}{c}{ F 28 d cu } \\
& A & B & C \\
\hline$K 1$ & 4.45 & 4.45 & 4.42 \\
$K 2$ & 4.76 & 4.73 & 4.55 \\
$K 3$ & 4.74 & 4.72 & 4.78 \\
$K 4$ & 4.44 & 4.48 & 4.64 \\
$R$ & 0.32 & 0.28 & 0.36 \\
\hline
\end{tabular}

flexural strength test were collected, this paper used the range method to analyze the data and study the three-dimensional placement of concrete with large size cobbles as well as two-dimensional mesoscopic numerical simulation of cracks.

\subsection{Three-Dimensional Placement of Concrete with Large Size} Cobbles. The two-graded aggregate for the four-point bending beam test was middle cobble: small cobble $=20 \%$ : $80 \%$, middle cobble: small cobble $=30 \%: 70 \%$, middle cobble: small cobble $=40 \%: 60 \%$, middle cobble: small cobble $=50 \%: 50 \%$. The aggregate sizes of middle cobbles and small cobbles were $40-20 \mathrm{~mm}$ and $20-5 \mathrm{~mm}$, respectively. The representative aggregate size was $30 \mathrm{~mm}$ and $12.5 \mathrm{~mm}$. The aggregate bulk density was $1.8 \times 10^{3}$. The density of cobbles in the specimen was $1219 \mathrm{~kg} / \mathrm{m}^{3}$. Therefore, we have the following.

Volume of the specimen $\mathrm{V}=0.00375 \mathrm{~m}^{3}$; weight of the cobble $=4.57 \mathrm{~kg}$; volume of the cobble $\mathrm{Vg}=2.54 \times 106 \mathrm{~mm}$.
The number of aggregates at each level of the simply supported beam was calculated and aggregates were randomly placed according to the data.

There is three-dimensional placement of concrete with large size cobbles in which the medium cobble: small cobble $=20 \%: 80 \%$.

The aggregate size of medium cobbles was $20-40 \mathrm{~mm}$. $D$ was $30 \mathrm{~mm}$. There was $n \times \pi \times D^{3} / 6=0.2 \times 2.54 \times 10^{6}$, and then the number of aggregates was $n=36$.

The aggregate size of medium cobbles was $5-20 \mathrm{~mm}$. $D$ was $12.5 \mathrm{~mm}$. There was $n \times \pi \times D^{3} / 6=0.8 \times 2.54 \times 10^{6}$, and then the number of aggregates was $n=1986$. Similarly, the number of aggregates can be obtained when the gradation is $70 \%: 30 \%, 60 \%: 40 \%$, and 50\%:50\%.

From Figure 3 shown, it can be found that the number of aggregates is different at different gradations. The results show that aggregates can be randomly generated and well distributed. The three-dimensional numerical simulation can well reflect the distribution of large size cobbles at different gradations through microanalysis.

2.5. Two-Dimensional Numerical Simulation of Cracks. The first principal stress effect in four-point bending beam for the concrete with large size cobbles at different gradations is shown in the following figure. This paper analyzed the middle section of the beam where the crack occurs. Results are as shown in Figures 4 and 5.

From the cloud diagram of the first principal stress, it can be seen that, under the same load, large size cobbles at 

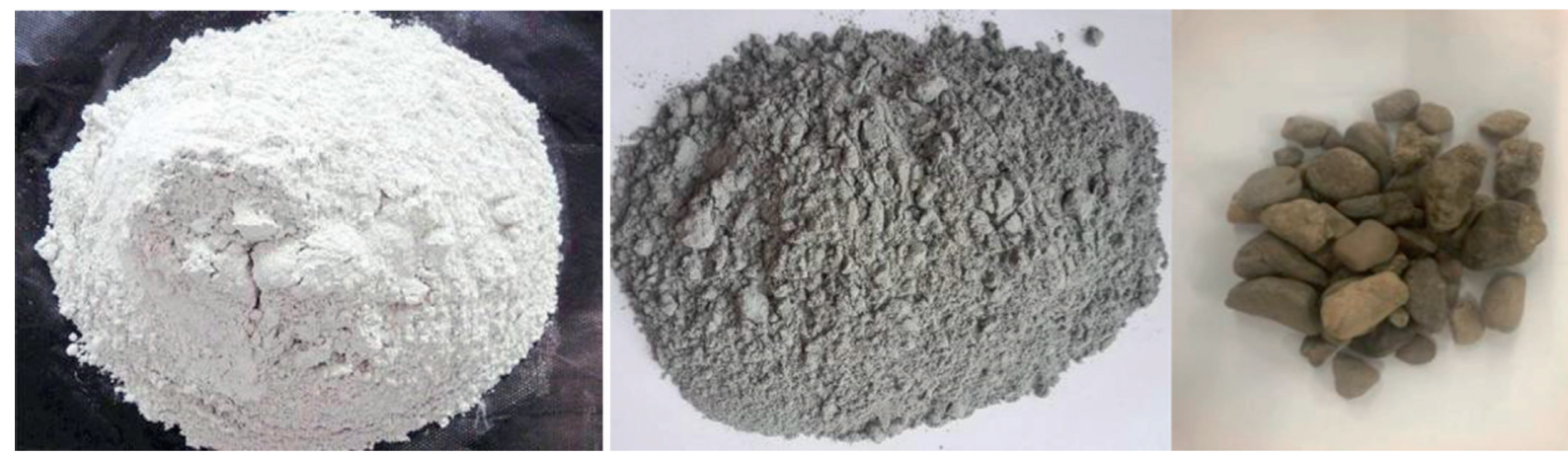

FIGURE 1: Slag powder, fly ash, and large size cobbles.

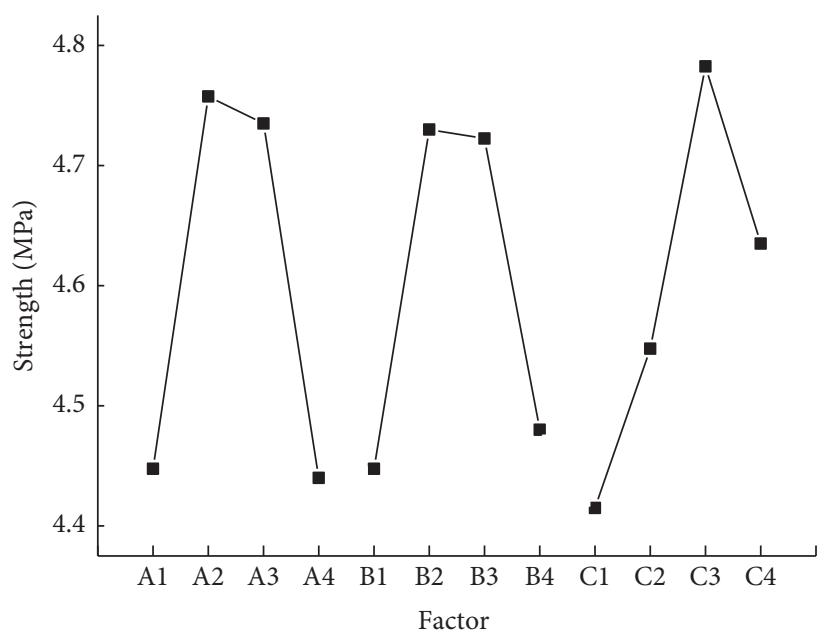

FIgURE 2: Main effect trend diagram for the flexural strength.

different gradations present different stress cloud patterns. With the increase of medium cobbles, the height of the crack increases first and then decreases. The cloud diagram of the stress is expanding along with the expansion of the crack. When the beam cracks under stress, the stress is released as the crack expands. Different heights of the crack generate different moire patterns, which are almost symmetrically distributed. When the gradation is $40 \%: 60 \%$, the cloud diagram of the stress on the four-point bending beam shows no obvious stress concentration. When the gradation is $20 \%$ : $80 \%$, the stress concentration of the concrete is the most obvious. And the higher the crack, the easier it is to reach the cracking load. With the increase of the load, the crack of the concrete becomes higher and gradually turns to the failure form.

2.6. Stress Distribution of the Concrete with Large Size Cobbles. In order to observe the stress distribution of the model clearly, we compared the stress contour of the concrete with cobbles at different gradations. Results are shown in Figure 6.

Figure 6 shows the stress distribution of the concrete with cobbles at different gradations. It can be seen that aggregates have loose contact. Under external forces, aggregates and the mortar squeeze mutually to form a force chain. The force chain basically shares the same direction as that of the stress. Elastic repulsive force will be formed between aggregates. When the repulsive force reaches a balance, the internal force chain will disappear. The distribution of strong force chains formed between aggregates can be clearly seen. The brighter and the darker the diagram of the force chain shows, the greater the stress is. The color of the diagram can clearly reflect the distribution of the internal stress.

According to the regional analysis of the stress, it is found that when the concrete is under the stress, the initial crack of the concrete is generated in the internal interface on both sides of the concrete, the bearing position at the bottom of the beam, and the load area at the upper end of the beam. It is easy for the specimen to produce extrusion force, which extends inward from the internal interface on both sides and the upper and lower ends. Then there is a crack in the middle of the concrete, and the stress is concentrated around the cobbles, where the concrete begins to crack around the aggregates. The sizes of nonforce chain area are different when aggregates have different gradations. According to Figure 6, when the gradation is $40 \%: 60 \%$, the nonforce chain area is the largest. There is the largest number of aggregates, which indicates that, at such gradation, 


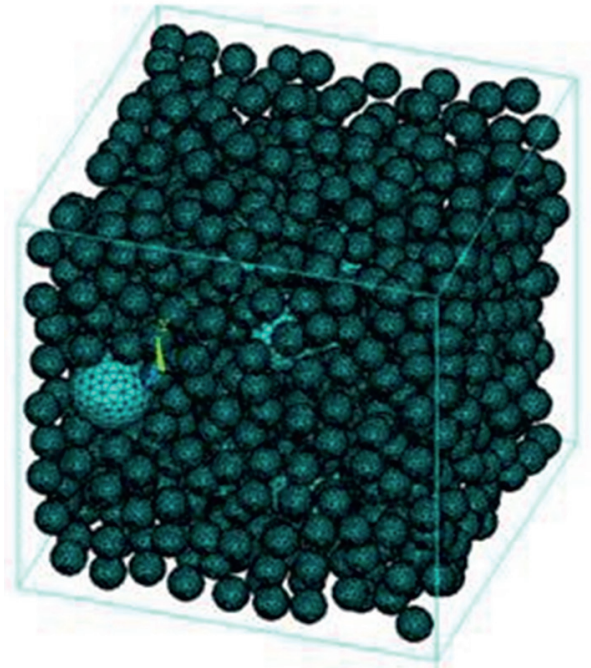

(a)

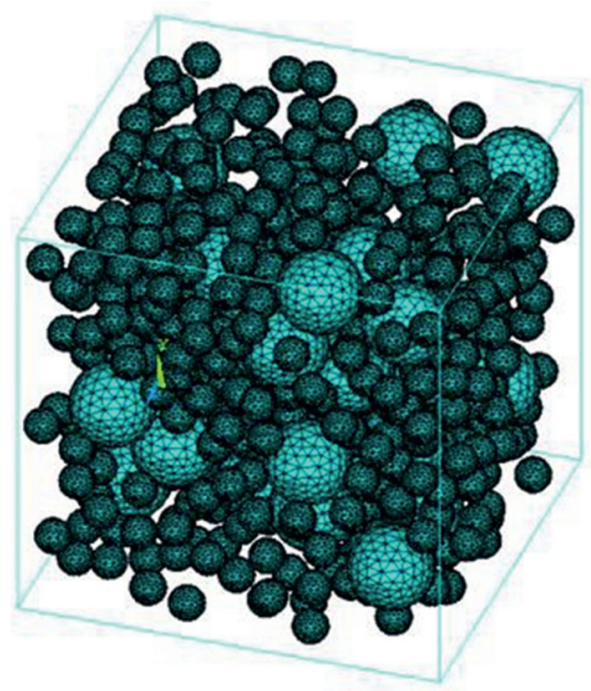

(c)

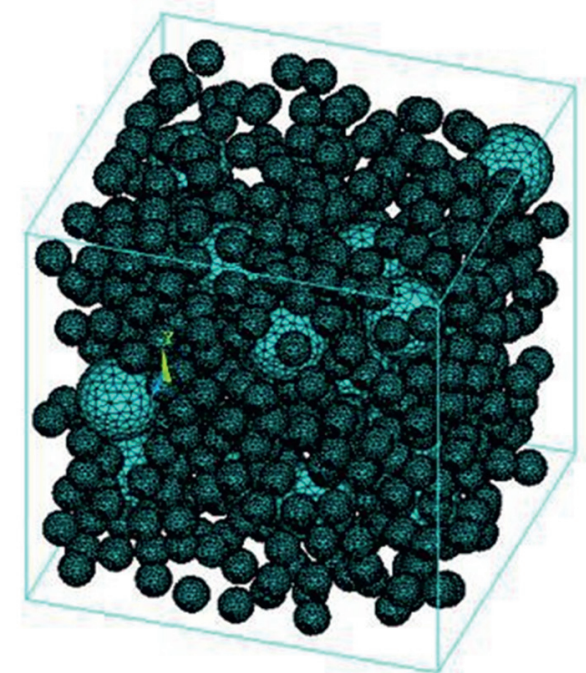

(b)

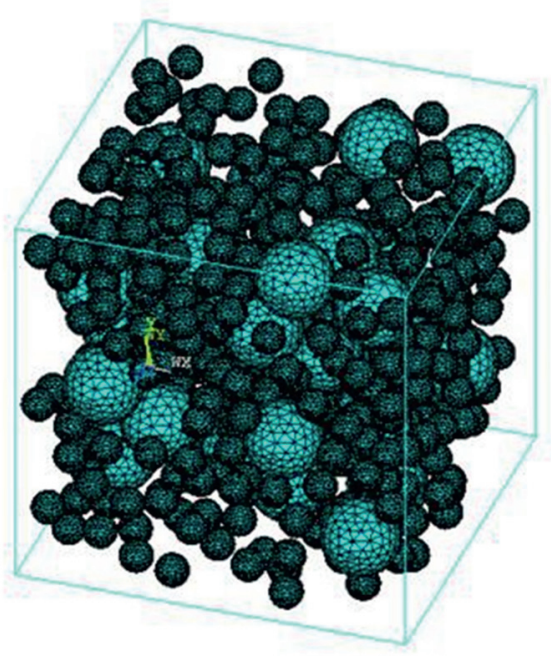

(d)

FIGURE 3: The three-dimensional microanalysis for the concrete with large size cobbles at different gradations: (a) 20\% : 80\%; (b) 30\% : 70\%; (c) $40 \%: 60 \%$; (d) $50 \%: 50 \%$.

aggregates produce a good bearing capacity and the flexural strength of the concrete reaches the highest. It can be seen from the figure that the stress contour for the concrete with large size cobbles at different gradation shows the same distribution.

Through the general postprocessing plate of ANSYS for the flexural strength test, we can obtain the displacement of the concrete with large size cobbles at different gradations in negative direction of $Y$-axis under ultimate load, as is shown in Figure 7. The cobble gradations are 20\%: $80 \%, 30 \%: 70 \%$, $40 \%: 60 \%$, and $50 \%: 50 \%$, respectively.

As is shown in Figure 7, it can be found that, with the increase of the load, the concrete with cobbles at different gradations displaces towards the same trend in the $Y$-axis. Both cobble and mortar bear the bending stress vertically, with the maximum stress occurring on the left and right side of the aggregates in the aggregate concentration area. They are arranged in horizontal strips. When the beam is under load, the heavier the load is, the larger the displacement in the direction of $Y$-axis is. When the load is applied, if there is stratification for the displacement, it indicates that cracks have started to develop, and there is macroscopic damage in the concrete, which means the concrete structure is no longer strong.

Given that the concretes with large size cobbles at different gradations show the same displacement, we choose 6 vertical nodes in the middle part of the cracked specimen at the gradation of $40 \%: 60 \%$ and define them as path A. We number the nodes $10189,3883,10235,4479$, 10884 , and 7684 as $1,2,3,4,5$, and 6 from the top to the bottom of the middle part of the specimen, as is shown in Figure 8.

The deformation values in the direction of $Y$-axis under 5, 20, 40, and 60 load substeps are listed in Table 4. 


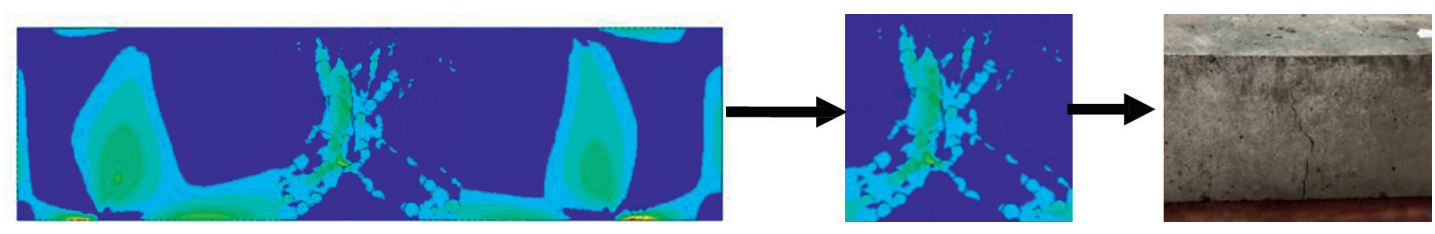

FIgURE 4: Middle section of the beam where the crack occurs.
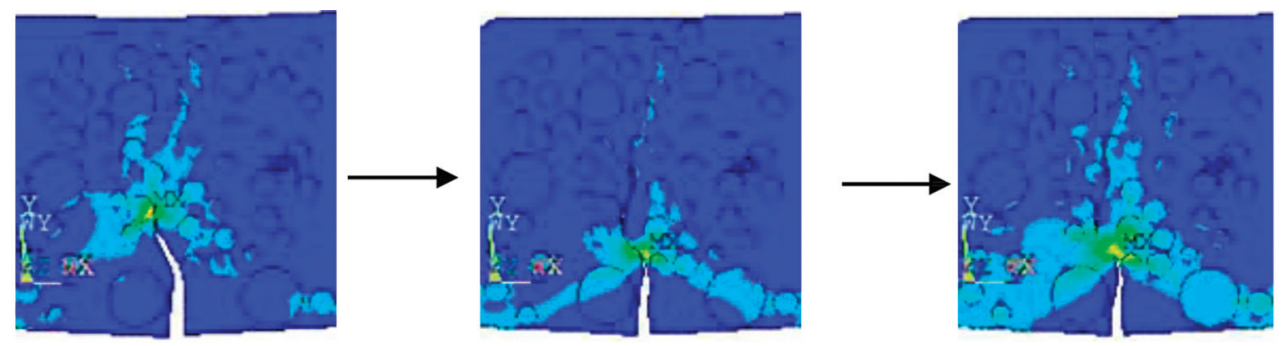

(a)
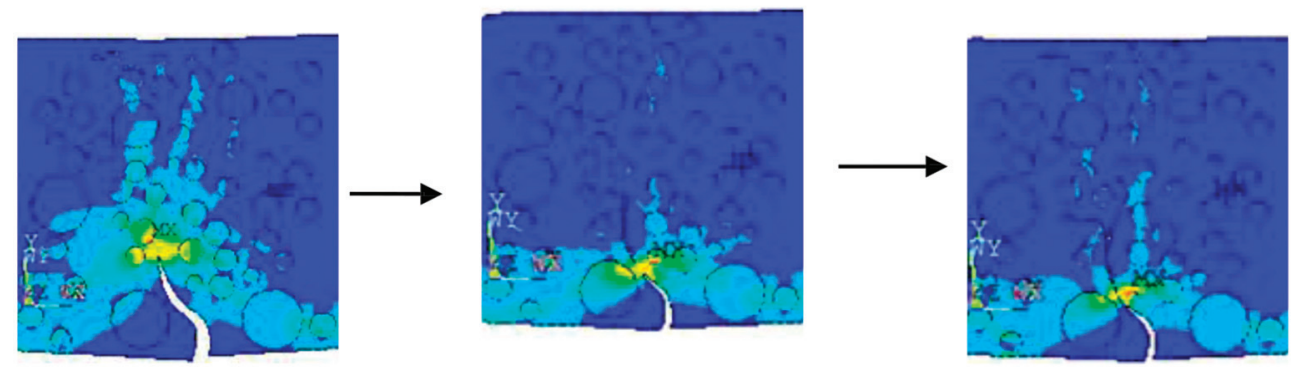

(b)
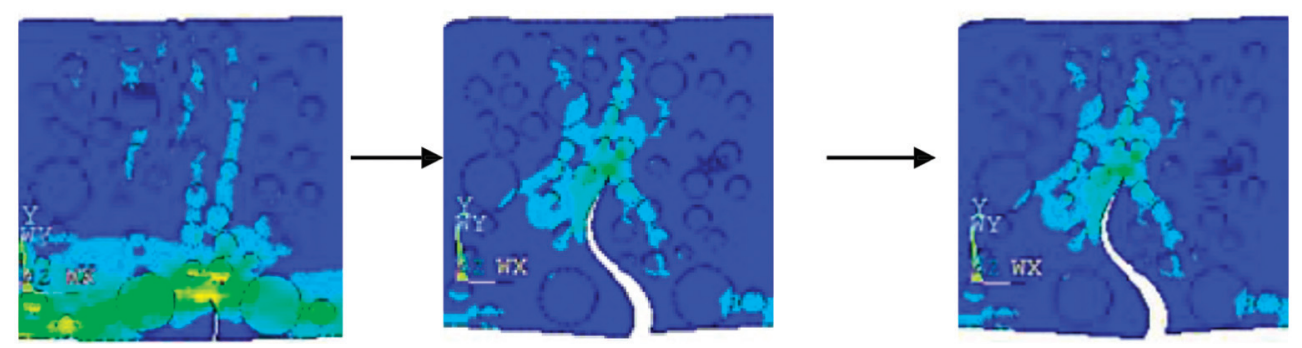

(c)
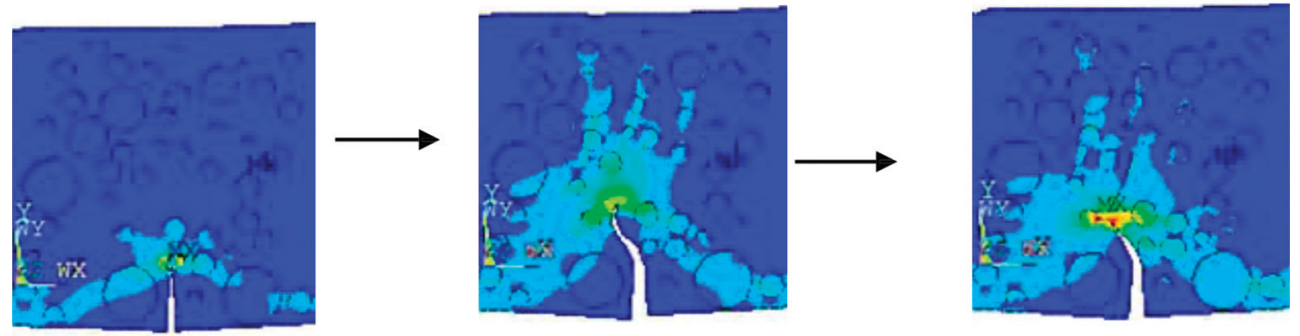

(d)

Figure 5: Concrete with large size cobbles at different gradations: (a) 20\%:80\%; (b) 30\%:70\%; (c) 40\%:60\%; (d) 50\%:50\%.

The deformation curves of 6 nodes under the 5th, 20th, 40th, and 60th load substeps are obtained, as is shown in Figure 9.

As is shown in Figure 9, with the increase of the load substep, the node becomes more deformed. The deformation of the nodes from under step 20 to step 60 is obvious. The second half nodes deform slowly. And the node 7684 always deforms more than other nodes. To study further, a new path is defined as B: The deformation curves of 6 nodes under different load substeps are shown in Figure 10.

From Figure 10, it can be seen that the nodes basically show the same deformation trend vertically. The 


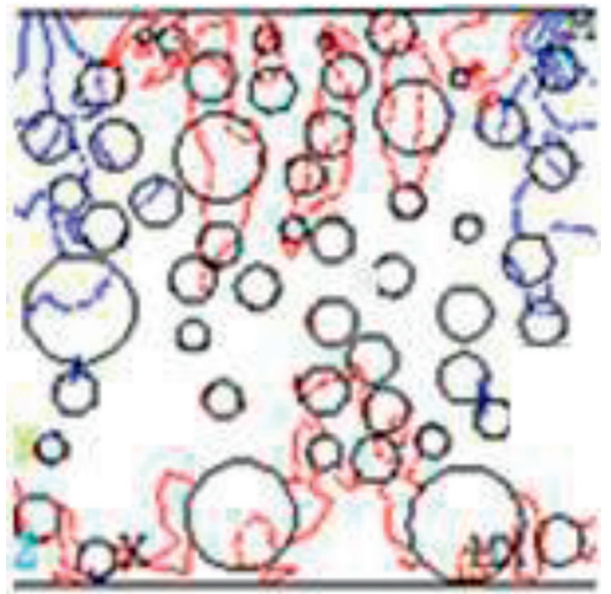

(a)

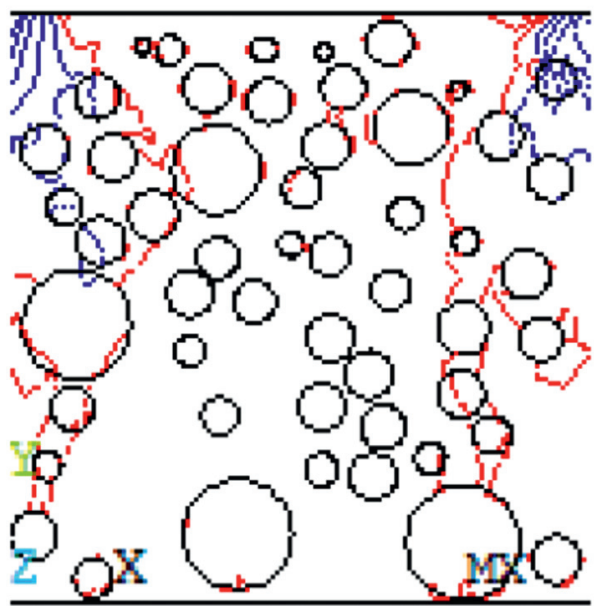

(c)

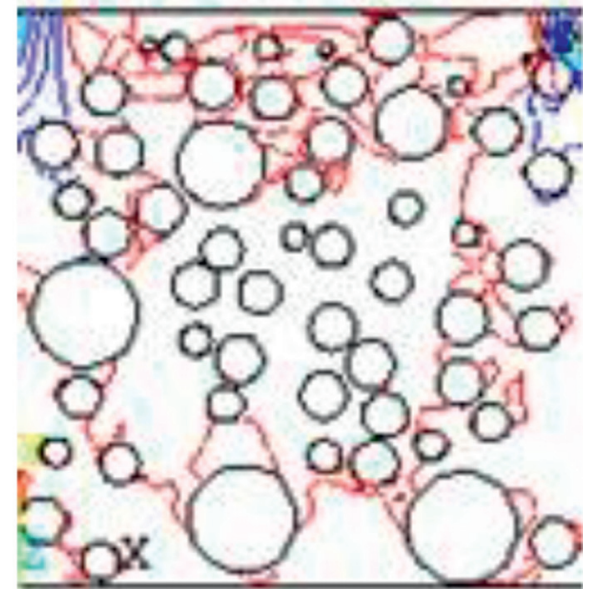

(b)

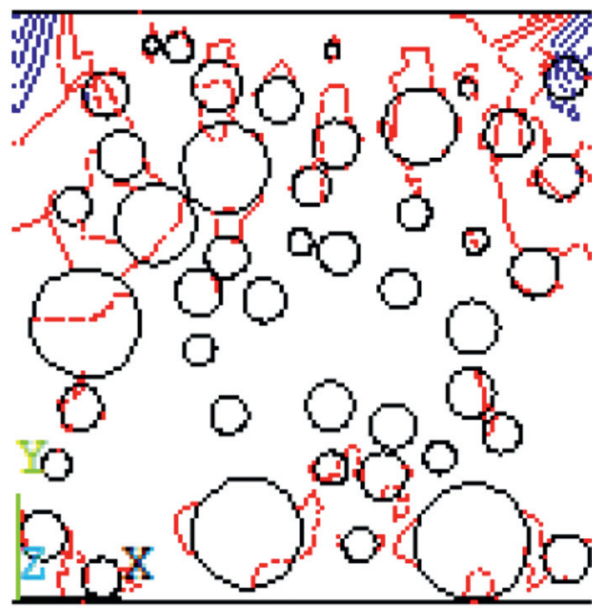

(d)

FIGURE 6: Stress contour of the concrete with cobbles at different gradations: (a) 20\%:80\%; (b) 30\%:70\%; (c) 40\%:60\%; (d) 50\%:50\%.

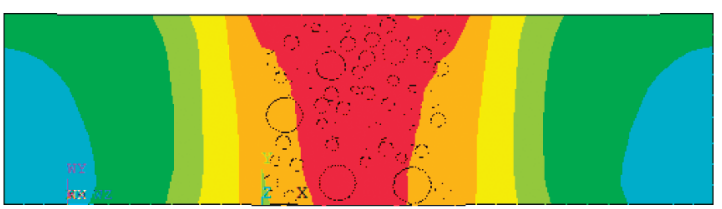

(a)

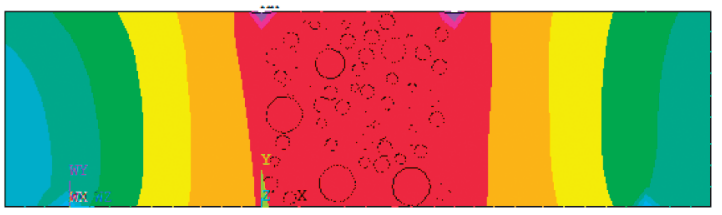

(c)

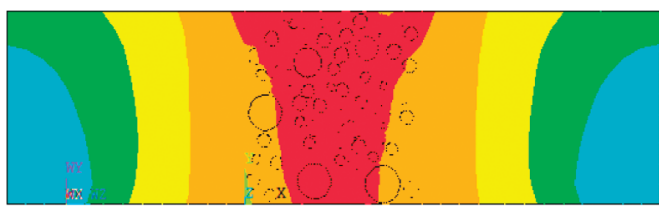

(b)

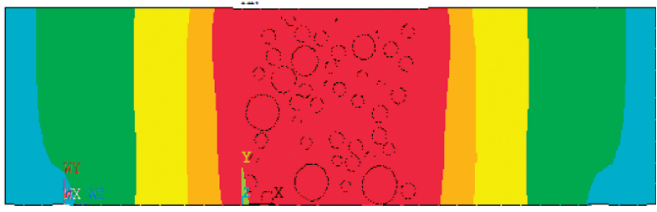

(d)

Figure 7: Displacement of the concrete in the direction of $Y$-axis.

deformation is obvious from under the 20th load substep to the 60th load substep where the crack initially takes shape. It shows that the heavier the load, the larger the displacement of the concrete in the direction of $Y$-axis.
After the simulation is completed, PRVAR command is used to export the stress-strain data to process. When the strain increases to a certain extent, although the concrete structure is not completely damaged, its bearing capacity is 


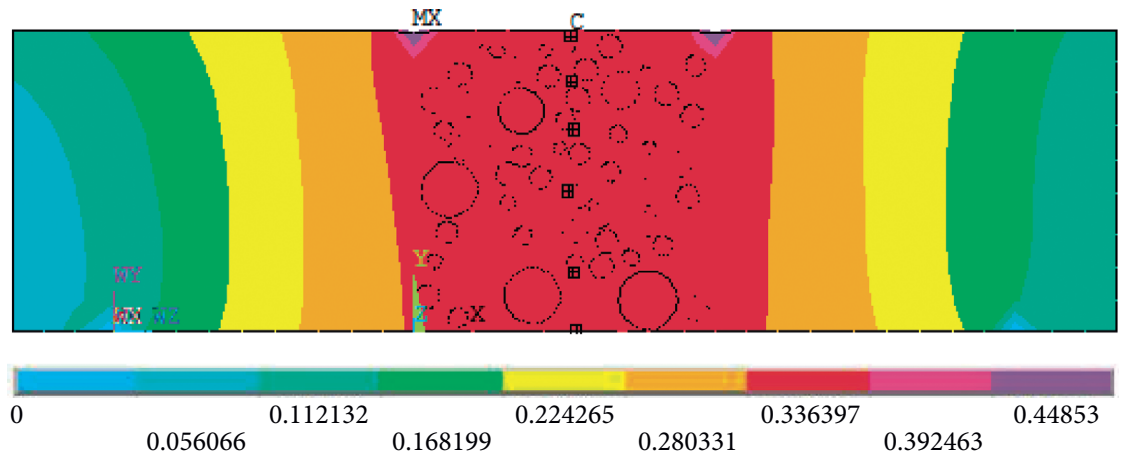

Figure 8: Diagram for path A.

TABle 4: Deformation values in the direction of $Y$-axis under different load substeps.

\begin{tabular}{lcccc}
\hline $\begin{array}{l}\text { Node } \\
\text { number }\end{array}$ & Step 5 load & $\begin{array}{c}\text { Step 20 } \\
\text { load }\end{array}$ & $\begin{array}{c}\text { Step 40 } \\
\text { load }\end{array}$ & $\begin{array}{c}\text { Step 60 } \\
\text { load }\end{array}$ \\
\hline 1 & 0.00433 & 0.01930 & 0.06420 & 0.10661 \\
2 & 0.03108 & 0.09183 & 0.13450 & 0.22367 \\
3 & 0.07358 & 0.17341 & 0.25863 & 0.31589 \\
4 & 0.10662 & 0.25513 & 0.30488 & 0.42505 \\
5 & 0.12776 & 0.27714 & 0.34814 & 0.47907 \\
6 & 0.15000 & 0.28410 & 0.39105 & 0.53290 \\
\hline
\end{tabular}

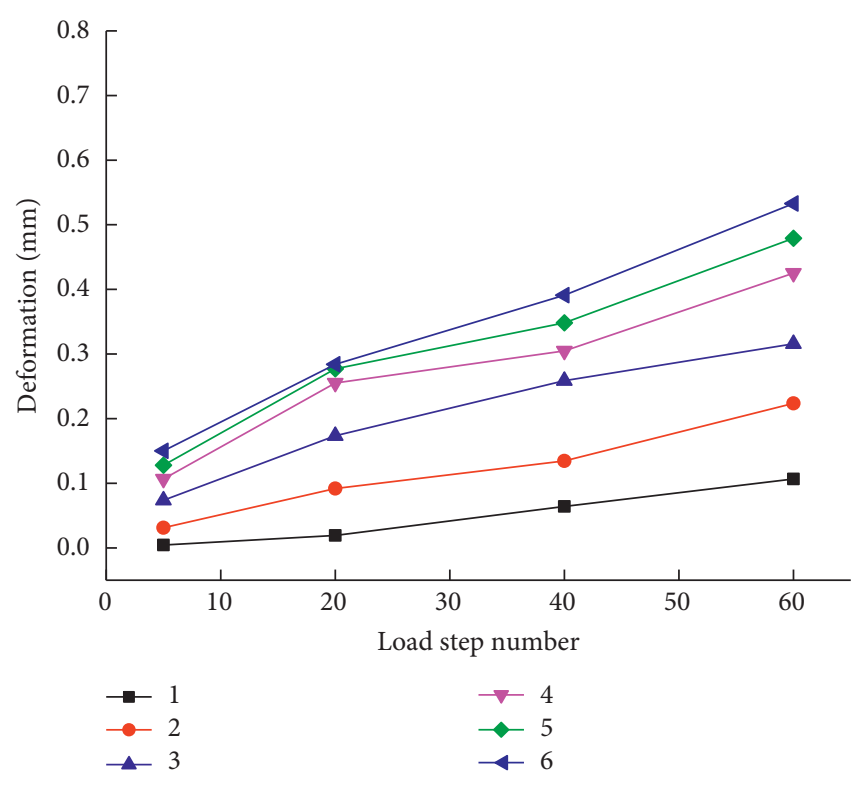

Figure 9: Node deformation on path A.

lost. Therefore, in order to observe how the stress-strain curve rises, we choose not to look at the smooth segment of the curve, as the falling segment is not defined in this paper. In the actual stress-strain curve, the ultimate load lasts for a short period of time. So we integrate four groups of data and display them on a single data map. Figure 11 shows the stress-strain curve of the concrete with large size cobbles at different gradations.

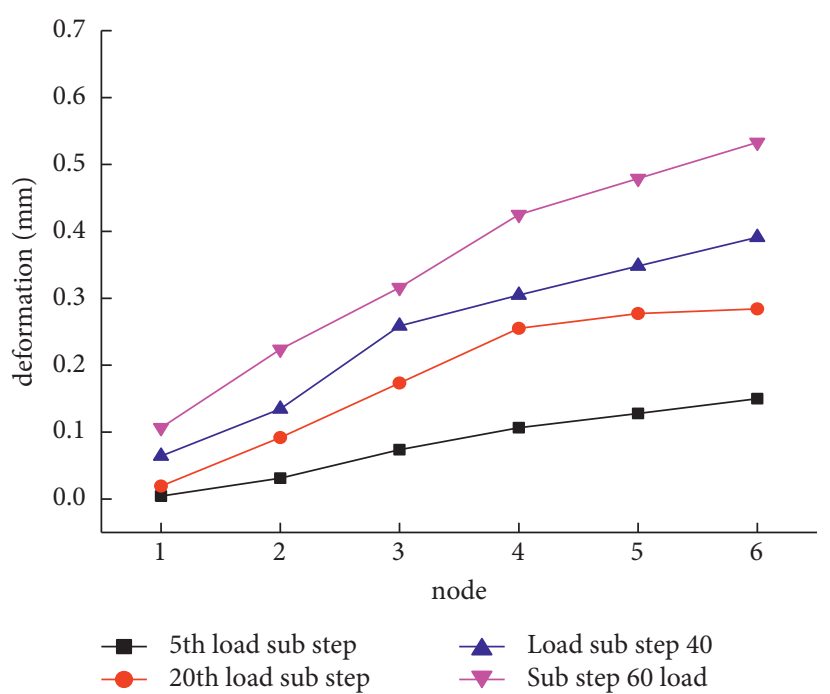

Figure 10: Node deformation on path B.

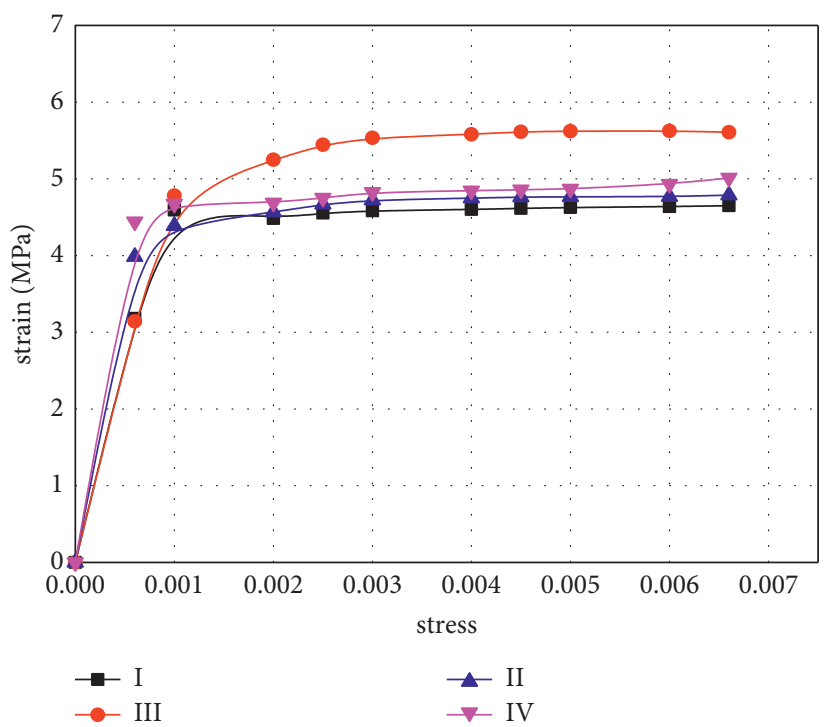

FIGURE 11: Stress-strain curve of the large sized cobble concrete at different gradations. 
From Figure 11, it can be seen that the flexural strength of the concrete with large size cobbles at different gradations follows the sequence: the concrete at the gradation of $40 \%$ : $60 \%$ has a flexural strength of $4.78 \mathrm{MPa}$; at the gradation of $50 \%: 50 \%$, $4.64 \mathrm{Mpa}$; at the gradation of $30 \%: 70 \%$, $4.55 \mathrm{MPa}$; and at the gradation of $30 \%: 80 \%, 4.42 \mathrm{MPa}$. From Figure 5, it can be seen that the ultimate strain of the concrete at four gradations is all around 0.001, indicating that the concrete is close to elastic failure.

As the stress-strain curve of ANSYS does not define the falling segment, when the stress-strain of the concrete reaches the ultimate strength, the curve tends to be horizontal, and the simulation results are consistent with experimental ones.

\section{Conclusions}

This paper analyzed the effect of the mixing amount of fly ash and slag and the gradation of large size cobbles on the performance of the concrete through macromechanics. Based on the basic theory of concrete numerical simulation, this paper used Monte Carlo method to establish a three-dimensional model and a two-dimensional model. And through ANSYS we simulated the crack of the concrete by microanalysis. This paper drew the following conclusions:

(1) The mechanical properties of the concrete first increase and then decrease with the total amount of slag and fly ash, and when the mixing amount of slag and fly ash is $10 \%$, the concrete has the largest flexural strength.

(2) The more compacting the aggregates and the mortar are filled, the less the crack of the concrete expands. The stress cloud diagram gradually extends upwards as the crack expands, and the stress is more concentrated.

(3) ANSYS shows that the sizes of nonforce chain area are different at different gradations of aggregates. When the gradation is $40 \%: 60 \%$, the nonforce chain area is the largest. There is the largest number of aggregates, which indicates that the gradation of aggregates produces a good bearing capacity and the flexural strength of the concrete reaches the highest.

(4) The gradation of cobbles affects the flexural strength of the concrete. It is recommended that follow-up studies further refine the cobble variables to make the numerical simulation more reasonable.

\section{Data Availability}

The data used to support the findings of this study are available from the corresponding author upon request.

\section{Conflicts of Interest}

The authors declare that there are no conflicts of interest regarding the publication of this paper.

\section{Acknowledgments}

This study was supported by the funds of Scientific Research Project of Colleges and Universities in Inner Mongolia Autonomous Region (NJZZ21013).

\section{References}

[1] Y. X. Zhang, Z. K. Lou, Z. Zhang, and Y. Y. Wang, "Influence of aggregate size effect on elastic modulus and strength of interfacial transition zone of concrete," Concrete, vol. 7, pp. 7-10, 2017.

[2] Y. Chen, X. Y. Han, X. Z. Hu, and Q. B. Li, "Design of concrete fracture property by average aggregate size," Cement and Concrete Composites, vol. 2021, Article ID 104105, 2021.

[3] M. Du, L. Jin, D. Li, and X. L. Du, "Meso numerical study on the influence of aggregate size on splitting tensile properties and size effect of concrete," Engineering Mechanics, vol. 34, no. 9, pp. 54-63, 2016.

[4] M. Mozaffar, A. K. L. Srivastava, and Z. Farooque, "Effect of varying size of aggregate on high strength concrete," International Research Journal of Engineering and Technology, vol. 8, no. 3, 2021.

[5] W. Zhong, J. Pan, J. Wang, and C. Zhang, "Size effect in dynamic splitting tensile strength of concrete: experimental investigation," Construction and Building Materials, vol. 270, Article ID 121449, 2021.

[6] P. Liu, "Research on properties of self-compacting concrete based on the characteristics of aggregate particles," Master's thesis, Northwest Sci-Tech University of Agriculture and Forestry, Xianyang, China, 2019.

[7] Q. C. Hao, "Effect of coarse aggregate particle gradation on the bending strength of concrete," Cement Engineering, vol. 2, pp. 89-91, 2018.

[8] Y. Liu, Z. Zhang, X. Liu, L. Wang, and X. Xia, "Ore image classification based on small deep learning model: evaluation and optimization of model depth, model structure and data size," Minerals Engineering, vol. 172, Article ID 107020, 2021.

[9] W. Zhang and Z. Tang, "Numerical modeling of response of CFRP-concrete interfaces subjected to fatigue loading," Journal of Composites for Construction, vol. 25, no. 5, 2021.

[10] H. Huang, M. Huang, W. Zhang, and S. Yang, "Experimental study of predamaged columns strengthened by HPFL and BSP under combined load cases," Structure and Infrastructure Engineering, vol. 17, no. 9, pp. 1210-1227, 2020.

[11] S. R. Kasu, S. Deb, N. Mitra, A. R. Muppireddy, and S. R. Kusam, "Influence of aggregate size on flexural fatigue response of concrete," Construction and Building Materials, vol. 229, Article ID 116922, 2019.

[12] J. G. Wu, "Discussion on the effect of coarse aggregate size on the workability, mechanical properties and durability of C80 concrete," Guangdong Building Materials, vol. 35, no. 5, pp. 14-16, 2019.

[13] T. Dittmer and H. Beushausen, "The effect of coarse aggregate content and size on the age at cracking of bonded concrete overlays subjected to restrained deformation," Construction and Building Materials, vol. 69, pp. 73-82, 2014.

[14] T. U. Mohammed and A. H. Mahmood, "Effects of maximum aggregate size on UPV of brick aggregate concrete," Ultrasonics, vol. 69, pp. 129-136, 2016.

[15] M. Karamloo, M. Mazloom, and G. Payganeh, "Effects of maximum aggregate size on fracture behaviors of self-compacting lightweight concrete," Construction and Building Materials, vol. 123, pp. 508-515, 2016. 
[16] S. K. Al-Oraimi, R. Taha, and H. F. Hassan, "The effect of the mineralogy of coarse aggregate on the mechanical properties of high-strength concrete," Construction and Building Materials, vol. 20, no. 7, pp. 499-503, 2006.

[17] J. Han, M. Zhao, J. Chen, and X. Lan, "Effects of steel fiber length and coarse aggregate maximum size on mechanical properties of steel fiber reinforced concrete," Construction and Building Materials, vol. 209, pp. 577-591, 2019.

[18] K.-M. Kim, S. Lee, and J.-Y. Cho, "Effect of maximum coarse aggregate size on dynamic compressive strength of highstrength concrete," International Journal of Impact Engineering, vol. 125, pp. 107-116, 2019.

[19] J. Guru Jawahar, C. Sashidhar, I. V. Ramana Reddy, and J. Annie Peter, "Effect of coarse aggregate blending on shortterm mechanical properties of self compacting concrete," Materials \& Design, vol. 43, pp. 185-194, 2013.

[20] X. H. Vu, L. Daudeville, and Y. Malecot, "Effect of coarse aggregate size and cement paste volume on concrete behavior under high triaxial compression loading," Construction and Building Materials, vol. 25, no. 10, pp. 3941-3949, 2011.

[21] B. X. Yuan, Z. H. Li, Z. L. Su, Q. Z. Luo, M. J. Chen, and Z. Q. Zhao, "Sensitivity of multistage fill slope based on finite element mode," Advances in Civil Engineering, vol. 2021, Article ID 6622936, 2021.

[22] B. X. Yuan, Z. H. Li, Y. M. Chen et al., "Mechanical and microstructural properties of recycling granite residual soil reinforced with glass fiber and liquid-modified polyvinyl alcohol polymer," Chemosphere, vol. 286, Article ID 131652, 2021.

[23] B. Bai, R. Zhou, G. Cai, W. Hu, and G. Yang, "Coupled thermo-hydro-mechanical mechanism in view of the soil particle rearrangement of granular thermodynamics," Computers and Geotechnics, vol. 137, no. 8, Article ID 104272, 2021.

[24] B. Yuan, Z. Li, Z. Zhao, H. Ni, Z. Su, and Z. Li, "Experimental study of displacement field of layered soils surrounding laterally loaded pile based on Transparent Soil," Journal of Soils and Sediments, vol. 21, no. 9, pp. 3072-3083, 2021.

[25] B. Bai, Q. Nie, Y. Zhang, X. Wang, and W. Hu, "Cotransport of heavy metals and $\mathrm{SiO} 2$ particles at different temperatures by seepage," Journal of Hydrology, vol. 597, Article ID 125771, 2021.

[26] J. Feng, G. Yin, H. Tuo, and Z. Niu, "Parameter optimization and regression analysis for multi-index of hybrid fiberreinforced recycled coarse aggregate concrete using orthogonal experimental design," Construction and Building Materials, vol. 267, Article ID 121013, 2021. 